\title{
LARGE-AREA CHARACTERIZATION OF URBAN MORPHOLOGY - MAPPING OF BUILT-UP HEIGHT AND DENSITY USING TANDEM-X AND SENTINEL-2 DATA
}

\author{
Christian Geiß ${ }^{1}$, Member, IEEE, Tobias Leichtle ${ }^{2}$, Michael Wurm ${ }^{1}$, Patrick Aravena Pelizari ${ }^{1}$, \\ Ines Standfuß ${ }^{1}$, Xiao Xiang $\mathrm{Zhu}^{3}$, Senior Member, IEEE, Emily So ${ }^{4}$, Stefan Siedentop ${ }^{5}$, \\ Thomas Esch ${ }^{1}$ and Hannes Taubenböck ${ }^{1}$ \\ ${ }^{1}$ German Remote Sensing Data Center (DFD), German Aerospace Center (DLR), 82234 Wessling- \\ Oberpfaffenhofen,Germany; christian.geiss@dlr.de, michael.wurm@dlr.de, patrick.aravenapelizari@dlr.de, \\ ines.standfuss@dlr.de, thomas.esch@dlr.de, hannes.taubenboeck@dlr.de \\ ${ }^{2}$ Company for Remote Sensing and Environmental Research (SLU), Kohlsteiner Straße 5, 81243 München, \\ Germany; tobias.leichtle@slu-web.de \\ ${ }^{3}$ Remote Sensing Technology Institute (IMF), German Aerospace Center (DLR) 82234 Weßling- \\ Oberpfaffenhofen, Germany; xiao.zhu@dlr.de \\ ${ }^{4}$ Cambridge University Centre for Risk in the Built Environment (CURBE), Department of Architecture, \\ University of Cambridge, 1-5 Scroope Terrace, Cambridge CB2 1PX, UK; ekms2@cam.ac.uk \\ ${ }^{5}$ ILS - Research Institute for Regional and Urban Development, Dortmund, Germany; stefan.siedentop@,ils- \\ forschung.de
}

\begin{abstract}
In this paper, we establish a novel multistep procedure for morphologic characterization of built environments in terms of built-up height and density. Thereby, we rely on elevation measurements from the TanDEM-X mission (TDM) and multispectral Sentinel-2 imagery. These earth observation systems feature a notable tradeoff between a fairly high spatial resolution and large-area coverage and, thus, allow for spatially continuous analysis of built environments around the globe. To this purpose, we follow an automated workflow which foresees the distinction of "built-up" and "non built-up" areas by relying on the so-called Global Urban Footprint processor (step 1). This information is deployed within a tailored filtering procedure for the TDM digital surface model data to extract elevation information for built-up areas (step 2). Subsequently, the intra-urban land cover is mapped under consideration of Sentinel-2 imagery and serves as basis to compute built-up heights and densities (step 3). These two measures are finally combined for a morphologic characterization of the built environment on an ordinal scale of measurement. Empirical validation efforts are provided based on comparative analysis with respect to more than 3.2 million individual building geometries and affiliated height measurements from cadastral data sources. The data sets cover the settlement areas of the capital cities and other major cities in Germany, England and the Netherlands. The experimental results underline the capability for a morphologic characterization of built environments with viable accuracies.
\end{abstract}

Index Terms - Sentinel-2, TanDEM-X, urban morphology, built-up height estimation, built-up density estimation

\section{INTRODUCTION}

Orara haracterization of built environments for large areas is a challenging but crucial task for e.g., monitoring change in the context of urbanization [1], [2], identifying specific settlement types [3], [4], analyzing population in a spatio- temporal manner [5], [6], evaluating the energy performance of different types of urban form [7]-[11], assessing vulnerability and risks with respect to natural hazards [12], [13], as well as quantifying urban heat islands [14], [15], among others.

To address the aforementioned applications, earth observation (EO; a table with all abbreviations used in this paper can be found in Appendix A) data were already identified as a valuable source of information. Regarding spatial resolution properties, past studies frequently relied on digital surface models (DSMs) (from e.g., LiDAR measurements) and optical imagery (from e.g., WorldView, GeoEye etc.) with a very high spatial resolution (VHR) to resolve and analyze the comparable small objects of built environments such as buildings (e.g., [16]-[18]). However, the deployment of VHR data hampers utilization capabilities due to availability, economic costs as well as processing requirements for large areas. When aiming at spatially continuous analyses and assessment approaches which are applicable for large areas such as nations, continents or even the globe, those kinds of data represent still a clear limitation nowadays.

However, especially recent EO systems internalize a remarkable tradeoff between a fairly high spatial resolution and large-area coverage. In particular, the TanDEM-X mission (TDM), which is a spaceborne radar interferometer, delivers a global DSM with an unprecedented pixel spacing of 0.4 arcseconds ( 12 meters) [19], [20]. Regarding optical imagery, ESA's Sentinel-2 satellites [21] deliver imagery with a spatial resolution of 10 meters for the bands covering visible light and near infrared. Thereby, Sentinel-2 data are provided free of charge to the public via a data hub. Given those properties, here, we complementarily employ data from both systems for large-area characterization of built environments. 
Regarding thematic resolution properties, past studies primarily characterized built environments with respect to building types [22]-[27], or urban structure types (i.e., distinctive and homogeneous assemblages of land cover/land use elements) [28],[29] with a high level of semantic detail based on sufficient and properly encoded prior knowledge. This allows inferring empirical relationships for predefined and specific semantic levels using supervised learning techniques (e.g., discriminate between buildings/zones of residential, commercial, and industrial usage).

In contrast to those approaches, we aim to quantitatively characterize built environments without the incorporation of prior knowledge and a priori determination of thematic classes according to specific semantics. This is done to allow for consistent and automated large-area analysis. Additionally, in this way, we bypass local idiosyncrasies (e.g., different settlements may contain different building types and similar types might feature a different physical appearance in various settlements, which is shaped by natural and cultural factors). With it, we aim for a consistent and objective statistical description of settlements. Thereby, such a quantitative characterization can be transferred into thematic classes $a$ posteriori and allows also for a targeted collection of in situ knowledge for dedicated applications.

Recently, Heinzel and Kemper [16] established an unsupervised workflow based on VHR airborne multispectral imagery for a joint description of settlements according to maximum building size, heterogeneity of the building size, and built-up density. To this purpose, they use operations from mathematical morphology [30] on the imagery. Based on spaceborne multispectral imagery, Zhang et al. [31] estimate building density in a supervised manner using advanced regression techniques. Gonzáles-Aguilera et al. [17] deploy VHR LiDAR data for derivation of geometric information (heights, areas, and volumes) and urban density attributes (building coverage ratio and floor area ratio) of buildings, land lots, and urban units. More thematically guided, Taubenböck et al. [18] produce three-dimensional building models in level of detail 1 (LoD-1) resolution (i.e., buildings are represented by extruded footprints) [32] by using a combination of VHR optical imagery and auxiliary data sources (such as geo-tagged ground photos or in situ surveys). Based on this data they characterize spatial patterns of buildings in terms of density, orientation and heterogeneity of alignment and compute individual building size and height for analysis of so-called "Arrival Cities".

However, as mentioned before and in contrast to previous works, we jointly exploit DSM data from the TDM and Sentinel-2 multispectral imagery to characterize urban morphology. These data sets feature a global coverage and allow for a unique mapping of urban morphology for large areas. At the same time, the spatial resolution properties of the data hamper analyses on individual building level. The pixel spacing of 0.4 arcseconds for the TDM data and 10 meters for the multispectral Sentinel-2 imagery can exceed the extent of the objects of interest (i.e., buildings). As a consequence, we work on an aggregated spatial level, i.e., we establish spatial processing units in terms of rectangular grid cells to compute morphologic properties of built-up structures. Thereby, we focus our work on the most constituent properties of urban morphology, namely built-up height and density. The latter represents one of the most important descriptive, explanatory as well as normative measures in urban research [33],[34]. In addition to that, the presence of buildings adds a third dimension to be considered among the environmental relationships found in urban areas. Thus, the vertical dimension of built environments must be taken into account to enable a holistic assessment [35]. Consequently, the measure built-up height is also incorporated in our approach. These two measures describing urban morphology are also in line with and can support generic mapping schemes such as the local climate zone (LCZ) concept [36], which aims to classify natural and urban landscapes into categories based on climaterelevant surface properties. Thereby, built-up height and density are determinant properties for categorizing urban landscapes [37], [38].

To address the aforementioned considerations, we establish a workflow to estimate built-up height and density for spatial processing units. These two measures are finally combined for a morphologic characterization of the built environment on an ordinal scale of measurement, which represents combinations of low, medium, and high built-up heights and densities. We built upon our initial works regarding this subject [39]. However, the contributions of this paper can be considered as follows.

1) A novel workflow is proposed to estimate built-up height and density automatically and derive corresponding ordinally scaled classes with respect to spatial processing units thereof. In particular, we first build upon the so-called Global Urban Footprint (GUF) processor [40]. This procedure provides binary information on "built-up" and "non built-up" areas on a global scale. It is deployed within a filtering procedure on the TDM DSM data to extract the required elevation information solely within built-up areas. Thereby, intra-urban water bodies are also automatically excluded. Subsequently, intra-urban land cover (LC) (i.e., LC within "built-up" areas as indicated by the GUF data set) is consecutively mapped according to the thematic classes "intra-urban vegetation", "elevated built-up", and "residual intra-urban LC". This information serves as basis to compute built-up heights and densities.

2) Exhaustive validation efforts are carried out in an original manner. We compute relevant accuracy measures based on comparisons with LoD-1 building models from areas which cover 10 large cities in three countries in Europe (i.e., Berlin, Hamburg, Munich, Cologne, Frankfurt, Stuttgart, London, Amsterdam, Rotterdam, and The Hague). As such, the main objective of this study is to evaluate the performance and overall suitability of automatic computations of built-up heights and densities from TDM and Sentinel-2 data for an efficient spatial differentiation of entire metropolitan regions into areas of low, medium, and high built-up height and density.

The remainder of the paper is organized as follows. Section II gives an overview of developed methods and section III is used to present the deployed data sets and explain the experimental setup. Section IV provides experimental results and validation efforts. Concluding remarks and an outlook are given in section $\mathrm{V}$. 


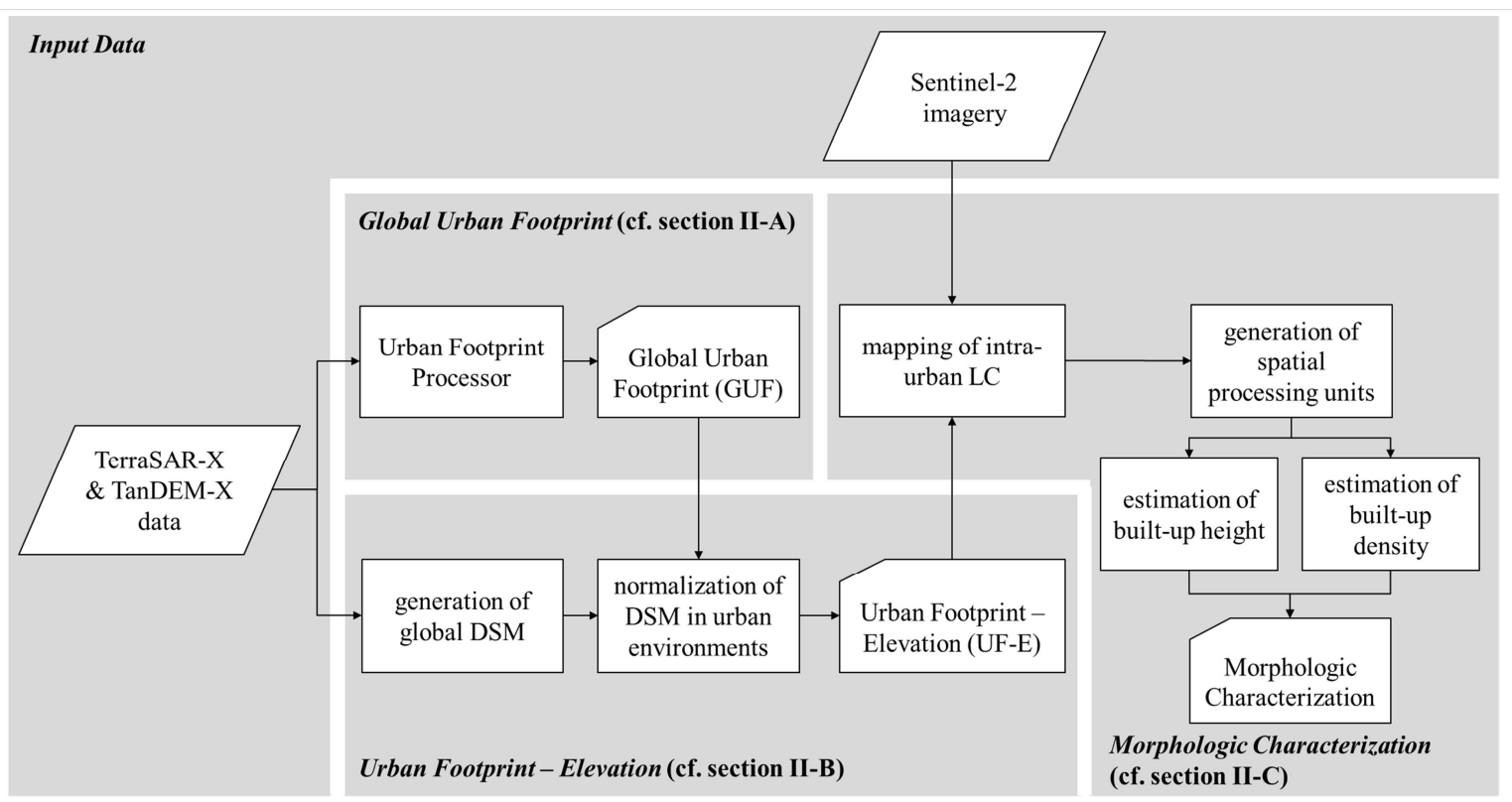

Fig. 1. Flowchart of the approach for characterization of urban morphology in terms of built-up height and density.

\section{PRoposed METHOdOLOGY}

A flowchart of the approach is given in Fig. 1. It builds upon data from the TDM and Sentinel-2 and consists of three main consecutive data processing modules. The first module builds upon the GUF processor, which is used to discriminate "built-up" and "non built-up" areas in a binary manner (Sec. II-A). The second module extends this binary description in terms of elevation information. To this purpose, elevation information in built environments is retrieved automatically using the DSM which was derived globally from the TDM (Sec. II-B). The third module contains the calculation of builtup height and density, which subsequently constitutes the generic morphologic characterization of built environments (Sec. II-C).

\section{A. Global Urban Footprint}

To efficiently constrain data processing and analysis on built environments, we build upon the outcomes of a fully automated image analysis procedure, which discriminates "built-up" and "non built-up" LC. Based on the TDM, which collected data sets of high resolution synthetic aperture radar (SAR) images, built-up areas can be extracted with an unprecedented spatial detail on a global scale. This is due to the circumstance that built-up areas show a distinct smallscale heterogeneity of local backscatter in the imagery. As such, the image analysis procedure foresees the computation of the speckle divergence as texture measure which is defined as the ratio between the local standard deviation and local mean of the backscatter computed in a defined local neighborhood. The speckle divergence will take very high values over built-up areas due to the occurrence of bright backscatter (as induced by e.g., double bounce effects and direct reflections at buildings) in close spatial proximity to dark areas (as induced by e.g., shadows or specular reflections at smooth surfaces such as roads). Subsequently, the speckle divergence is jointly deployed with the original backscattering amplitude for classification of built-up areas. To this purpose, the classification procedure first implements thresholds derived from image statistics of the amplitude and texture data, respectively, to reliably identify image elements (i.e., pixels) of built-up areas. The identified image elements serve as labeled samples for learning one-class classification models based on Support Vector Domain Description to establish optimal model solutions for the individual image scenes [40].

Generally, the data set does not contain spatially and thematically exact measurements of individual urban objects, but provides a more abstract delineation of the physical manmade properties of cities (i.e., built-up areas). Finally, postediting procedures ensure an overall absolute accuracy of about $85 \%$ [40], which underlines a reliable identification of built-up areas [41].

\section{B. Urban Footprint - Elevation}

In combination with the GUF data set, the elevation measurements of the TDM are used to compute a normalized DSM (nDSM). Consequently, the latter comprises elevation information of objects above ground in built-up areas. To this purpose, a digital terrain model (DTM) is derived first from the DSM with a so-called region growing-based progressive morphological filter procedure. This approach was first proposed by Geiß et al. [42] to address general challenges associated with the use of morphological filters in non-flat terrain and is intended to overcome particular challenges related to the spatial resolution of TDM data.

A detailed description of the underlying algorithms with pseudocode is provided in [42]. The procedure includes a multistep method using concepts of morphological image filtering, region growing, and interpolation techniques. It is based on the idea of progressive morphological filters, which aim to discriminate ground and non-ground pixels in the DSM based on algebraic set operations. Such filters identify nonground pixels in the DSM by gradually increasing the size of a structuring element and applying iteratively an elevation difference threshold. After the identification of initial nonground pixels, here, potential non-ground pixels are identified 
within each iteration and their similarity with respect to neighboring non-ground pixels is assessed. Potential nonground pixels are finally labeled as non-ground if they feature a high similarity to already identified non-ground ground pixels. Thereby, it is also made sure that intra-urban water bodies are excluded from the analysis using the TDM water indication mask, which is an automatically processed information layer included in the TDM DSM product [43].

After complete identification of non-ground pixels, corresponding ground pixels are subsequently interpolated to a DTM using an exact interpolation technique. Finally, the DTM is subtracted from the DSM to receive the final nDSM in built environments (i.e., Urban Footprint - Elevation (UFE)).

\section{Morphologic Characterization}

The calculation of built-up height and density is based on an intra-urban LC map. To establish the LC map, the UF-E data set is combined with Sentinel-2 imagery. The latter serves for computing the Normalized Difference Vegetation Index (NDVI) [44]. The NDVI internalizes different reflection properties of vegetation in the red and NIR band, respectively. High numerical values indicate photosynthetically active vegetation. In this manner, it can be noted that the GUF algorithm relies on distinctive SAR-related backscatter mechanisms of vertical, unpenetrateable structures (i.e., builtup structures) and, thus, the data set does not contain larger fractions of vegetation (e.g., urban forests [45]) [46]. However, small vegetation patches are automatically excluded before further processing by adaptively thresholding NDVI values of Sentinel-2 imagery using Otsu's method. The method searches for a threshold to separate two classes with minimum intra-class variance, i.e., maximum inter-class variance [47]. In this method, the group with higher NDVI values corresponds to intra-urban vegetation, whereas the group with lower NDVI values contains the remaining LC classes. Subsequently, elevated built-up areas are discriminated from residual intra-urban LC. This is done to prune intra-urban LC which cannot represent the objects of interest (i.e., buildings). Thereby, we assume that pixels within the residual settlement area (i.e., built-up areas without intraurban vegetation as identified in the previous processing step) represent elevated built-up areas if they exceed a certain height threshold $\Theta$ in the associated UF-E model.

The actual calculation of built-up height and density as well as their joint representation for final morphologic characterization is provided on spatial processing units. This is done to allow for a robust representation of extracted height values based on a statistical measure of central tendency (i.e., a quantile $Q_{n}$ ) and computing densities from the binary elevated built-up area pixels. In addition, in this way, robust comparisons to reference data with different resolution properties can be achieved. Here, rectangular grid cells of size $\mathrm{a} \times \mathrm{a}$, based on the arrangement of the image elements of the Sentinel-2 data, are used to rely on comparable spatial entities which are ubiquitously available throughout built environments (alternatively, irregular spatial processing units such as superpixels from an image segmentation procedure [29] or street blocks generated from geospatial vector data

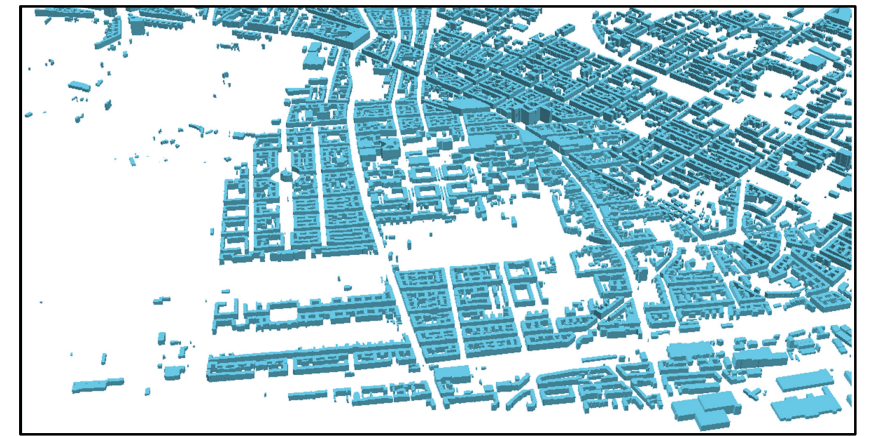

Fig. 2. LoD-1 model for a subset of the city of Berlin.

[25] can be exploited). Consequently, built-up height and density per grid cell are calculated as follows:

$$
\begin{aligned}
& \text { built - up height } t_{\text {grid cell }} \\
& \quad=Q_{n}\left(n D S M_{\text {elevated built-up }}\right)
\end{aligned}
$$

where $Q_{n}$ is the aggregation function (i.e., statistical measure of central tendency) and $n D S M_{\text {elevated built-up are the }}$ numerical height values contained in the UF-E model for the pixels labeled as LC class "elevated built-up";

$$
\text { built }- \text { up density } \text { grid cell }=\frac{A_{\text {elevated built-up }}}{A_{\text {built }-u p}}
$$

where $A_{\text {elevated built-up }}$ is the area covered by pixels labeled as LC class "elevated built-up" and $A_{\text {built-up }}$ is the area covered by pixels labeled as "built-up" (i.e., GUF).

Finally, estimated built-up height and built-up density values per grid cell are joined to nine morphologic classes which represent combinations of low, medium, and high builtup heights and densities.

\section{DATA SETS AND EXPERIMENTAL SETUP}

\section{A. DSM Data from TanDEM-X}

As mentioned earlier, the TDM elevation model can be dominantly regarded as a DSM, especially when analyzing built environments as in this study. Only few surfaces such as ice, snow, or vegetation can be penetrated by the X-band SAR signal [48]. Comparisons to ICESat data underline the high quality of elevation measurements, which feature less than one meter deviation in absolute vertical accuracy for surfaces other than highly vegetated areas or snow-/ice-covered regions. [49]. Overall, $14 \mathrm{TDM}$ tiles $\left(1^{\circ}\right.$ by $\left.1^{\circ}\right)$ with a spatial resolution of 0.4 arcseconds (i.e., $\sim 12$ meters) were processed to consistently cover the settlement areas of all 10 considered cities.

\section{B. Multispectral Imagery from Sentinel-2}

The multispectral Sentinel-2 imagery were subject to atmospheric corrections within the Sentinel Application Platform [50] using the Sen2Cor module [51] to provide level 2A products. We deploy Sentinel-2's red (665 nm) and nir (842 nm) bands, which feature a spatial resolution of 10 meters, for computation of NDVI values. The imagery for the different cities was acquired in autumn and winter of the years 2014-2016. The dates were chosen to reduce the effect of vegetation on the intra-urban LC classification since intra- 
urban vegetation frequently obscures underlying built-up structures [52].

\section{Reference Data}

As reference data sets, we incorporated LoD-1 building models comprising more than 3.2 million building geometries and affiliated height measurements, which are based on cadastral information for the cities in Germany [53] and the Netherlands [54], as well as edited OpenStreetMap data for the city of London [4]. Fig. 2 illustrates the reference LoD-1 models for a subset of the city of Berlin (the subset corresponds also to the area which is shown in the zoom-in windows of Fig. 4). The building geometries were resampled and properly aligned to the image elements of the Sentinel-2 data for comparison. The resulting binary built-up mask was used for computation of built-up height and density per grid cell, whereas median height values (i.e., $Q_{50}$ ) were deployed for subsequent comparisons.

\section{Experimental Setup and Parameterization}

The filter procedure for normalization of the DSM needs some free parameters to be fixed. The structuring element of the morphological filter must always exceed a building's outline to ensure extraction of all buildings present in the area under investigation. Consequently, the side length of the structuring element was determined empirically for the study areas according to the largest buildings present in the data. Additionally, an elevation difference threshold and a similarity constraint need to be determined. Both were set in accordance to previous experimental analysis, to enable a favorable tradeoff between decrease of omission errors and increase of commission errors when classifying ground pixels (i.e., $2.6 \mathrm{~m}$ for the elevation difference threshold and $1 \mathrm{~m}$ for the similarity constraint [42]). Besides, we derived a binary water mask with maximum extent from the water indication mask, which is provided with the TDM data, by selecting values from 3 to 127 (i.e., thresholds of TDM amplitude and coherence) [43]. Inverse distance weighting [55] with an adaptive neighborhood was deployed for interpolation of identified ground pixels. The deployed GUF data sets feature a spatial resolution of $12 \mathrm{~m}$. The obtained scene-specific NDVI thresholds, as determined with Otsu's method, are quite restrictive in classifying vegetation and range from 0.35 (Munich) to 0.46 (Cologne). We evaluated results as a function of different height thresholds for establishing the intra-urban LC map, i.e., $\Theta=\{>0 \mathrm{~m}, 3 \mathrm{~m}, 5 \mathrm{~m}\}$. In addition, we tested different aggregation functions for estimation of built-up height per grid cell. As described before, we represent height values of grid cells by quantiles $Q_{n}$. For a systematic evaluation, different deciles are tested, i.e., $Q_{50}, Q_{70}, Q_{90}$. Previous experiments showed that built-up heights are in tendency underestimated [39]. This motivated us to also establish built-up height estimations using upper deciles in addition to the median to eventually balance likely underestimations. Regarding the edge length of grid cells, we evaluate results with respect to $a=\{200 \mathrm{~m}, 500 \mathrm{~m}, 800 \mathrm{~m}\}$. Those values were found empirically to allow reflecting areas of homogeneous urban morphology in previous studies (e.g., [4], [34]). As described earlier, the measurements are finally combined within the defined nine morphologic classes. The thresholds for low, medium, and high built-up heights and densities were set according to numeric values, which are consistent with the values provided by the LCZ concept [36], i.e., a threshold of 0.2 for low built-up densities and 0.4 for high built-up densities was chosen, in conjunction with a threshold of $10 \mathrm{~m}$ for low built-up heights and $25 \mathrm{~m}$ for high built-up heights, respectively.

Statistical evaluation is carried out based on a set of accuracy measures. The accuracy of built-up height and density estimates is evaluated based on mean error (ME):

$$
\mathrm{ME}=\frac{1}{\mathrm{~N}} \sum_{\mathrm{i}=1}^{\mathrm{N}}\left(\widehat{\mathrm{X}}_{\mathrm{i}}-\mathrm{X}_{\mathrm{i}}\right),
$$

where $\widehat{X}_{i}$ is the numerical value per grid cell computed from the intra-urban LC map $\widehat{X}, X_{i}$ is the numerical value per grid cell derived from the reference data $\mathrm{X}$, and $\mathrm{N}$ is the number of grid cells. Thereby, positive ME values (i.e., $\mathrm{ME}>0$ ) indicate
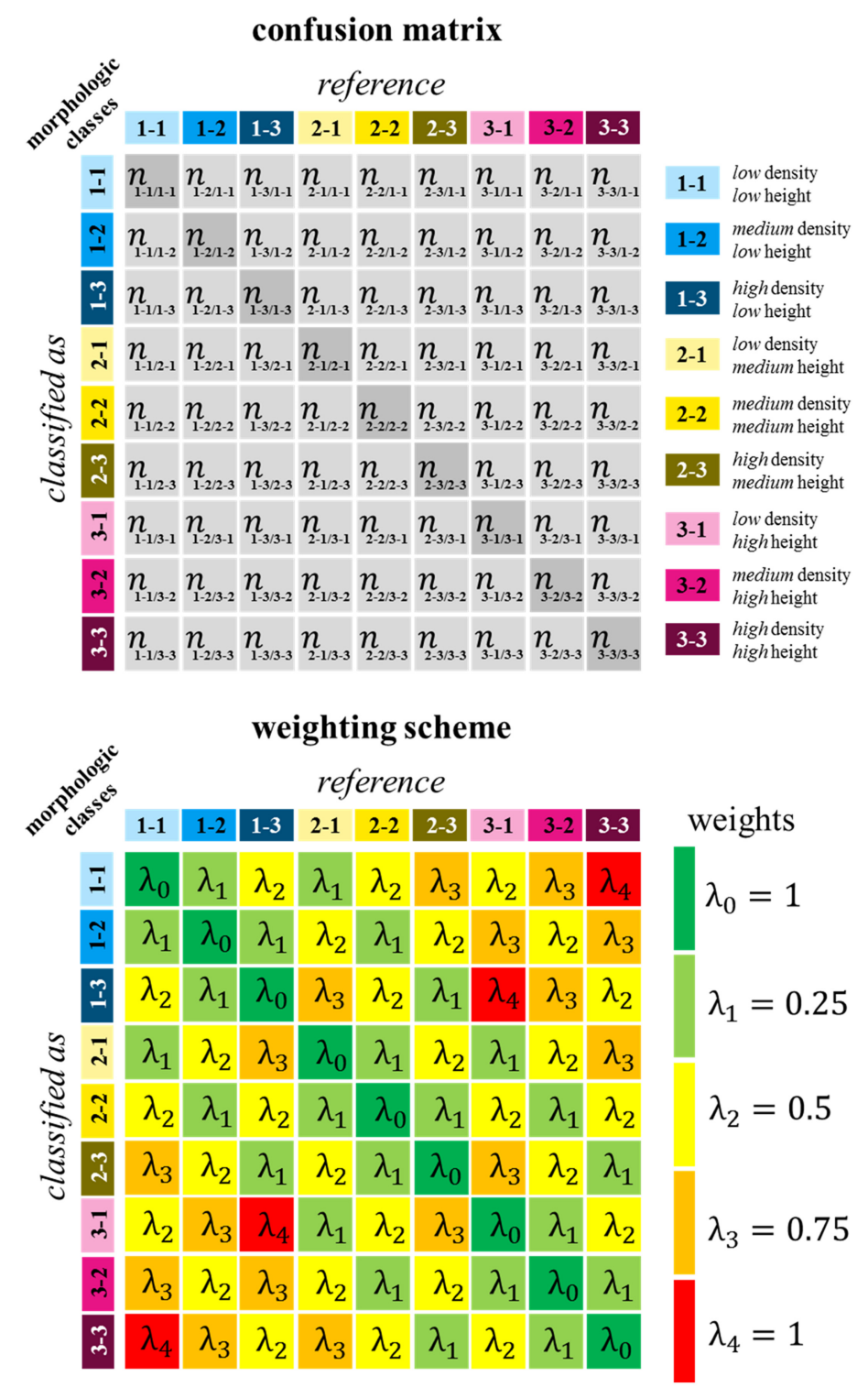

Fig. 3. Confusion matrix and weighting scheme with respect to the nine ordinal morphologic classes for computing a weighted confusion matrix. 
an overestimation, whereas negative $\mathrm{ME}$ values (i.e., $\mathrm{ME}<0$ ) indicate an underestimation of built-up height and density estimates with respect to the reference. In addition, the mean absolute error (MAE) is computed analogously [56] to provide absolute deviation levels:

$$
\mathrm{MAE}=\frac{1}{\mathrm{~N}} \sum_{\mathrm{i}=1}^{\mathrm{N}}\left(\left|\widehat{\mathrm{X}}_{\mathrm{i}}-\mathrm{X}_{\mathrm{i}}\right|\right) .
$$

Regarding the final combination of computed built-up height and density within nine morphologic classes, we derived classification accuracy measures from a weighted confusion matrix to account for the ordinal scale of measurement, which prohibits the valid use of accuracy measures derived from a conventional confusion matrix [57]. Consequently, a deviation of one ordinal class (e.g., class "low density - low height" is confused with "low density - medium height") is solely penalized with a weighting factor of 0.25 , two classes are penalized with a factor of 0.5 , three classes are penalized with a factor of 0.75 , and higher deviations are fully penalized. For illustration, Fig. 3 shows the weighting scheme for the number of instances $n$ of the respective morphologic classes.

This scheme is multiplied with the unweighted confusion matrix, whereby the residual instances, i.e., $((1-\lambda) * n)$ are added to the correctly classified instances of the respective class located at the diagonal of the matrix. To evaluate classspecific differences in accuracy, we derived user's and producer's accuracies from the weighted confusion matrix. In addition, overall accuracy (OA) as well as $\kappa$ statistic [58] were computed as global accuracy measures.

\section{EXPERIMENTAL RESULTS AND DISCUSSION}

An exemplary visualization of the outcomes of the different data processing steps in terms of TDM DSM data with binary water mask, atmospherically corrected Sentinel-2 data, GUF, and UF-E for the city of Berlin, Germany, is provided in Fig. 4. In addition, the intra-urban LC map is also shown, which serves as a basis to compute built-up height and density. The zoom-in window already allows inferring some fundamental properties of the respective data sets. The TDM DSM resolution of $\sim 12$ meters does not consistently allow reconstructing the individual building footprints. However, the built-up structures can be clearly depicted in the grayscale image representation of the DSM (Fig. 4a). To extract complementary information, multispectral Sentinel-2 imagery offers a suitable trade-off between covering large areas and simultaneously capturing details of the built environment (Fig. 4b). The GUF data set accurately identifies "built-up" LC, whereby larger vegetation areas or bare soil patches between built-up areas are also correctly labeled as "non built-up" (Fig. 4c). The intra-urban nDSM (i.e., UF-E) extends the thematic resolution of the GUF data sets by assigning a rational scaled elevation value, which also clearly allows identifying elevated objects of built environments (Fig. 4d). To further refine the spatial distinction of "built-up" and "non built-up", intra-urban vegetation areas are labeled under consideration of the multispectral imagery (Fig. 4e). Subsequent to that, the UF-E model is used to further distinguish between "elevated builtup" and "residual intra-urban LC" in the remaining areas $(\Theta$ was set to three meters in this visualization, which corresponds approximately to one floor [59]). Only pixels of the class "elevated built-up" and affiliated elevation values from the UF-E model are used for mapping built-up heights and densities and creating the morphologic classes. 

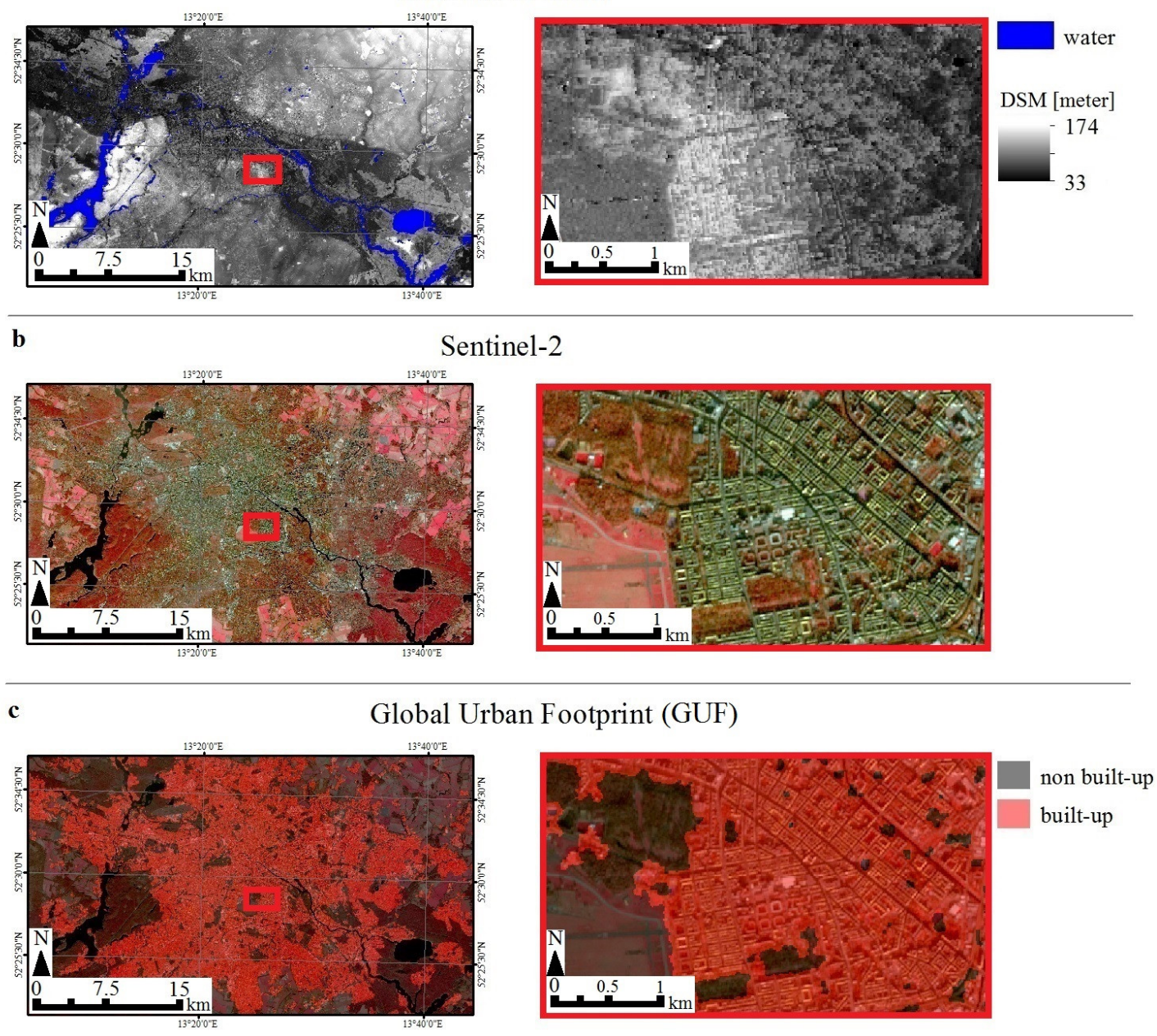

d

Urban Footprint - Elevation (UF-E)
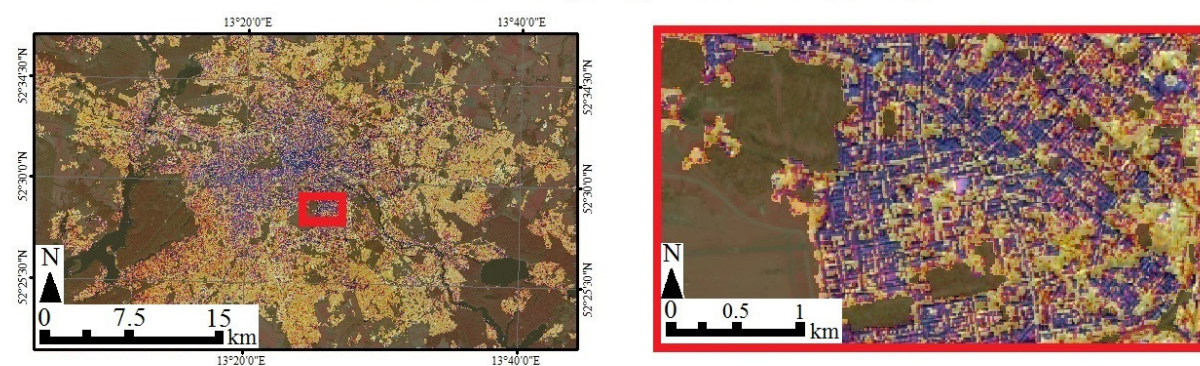

non built-up nDSM [meter]
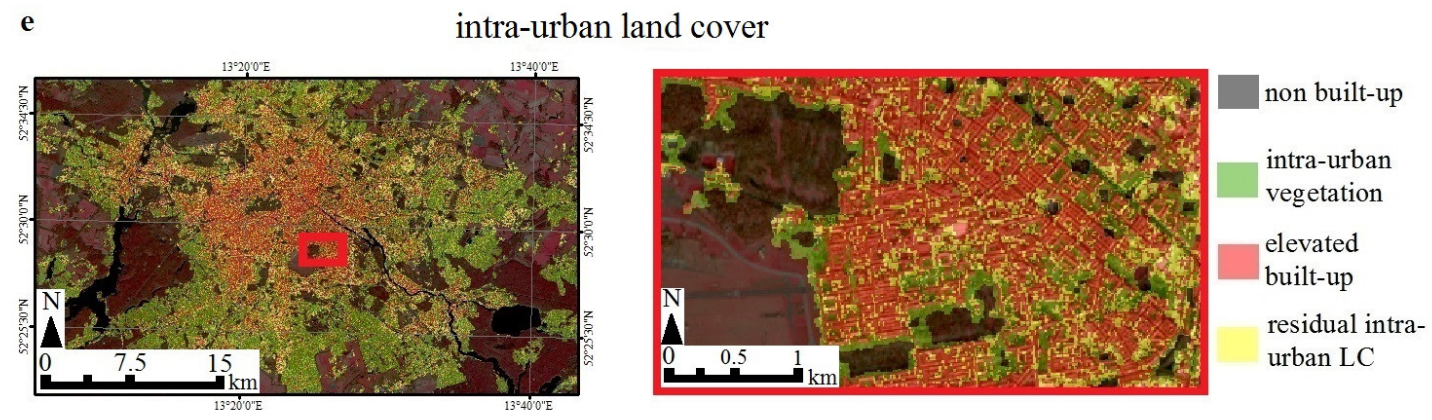

Fig. 4. Overview on the different data sets for the city of Berlin, Germany. (a) TDM DSM data with binary water mask. (b) Atmospherically corrected Sentinel-2 multispectral imagery in false-color representation. (c) GUF data set which discriminates "built-up" areas from "non built-up" areas. (d) The UF-E model provides intra-urban elevation values. (e) Intraurban LC map which discriminates three thematic classes. 


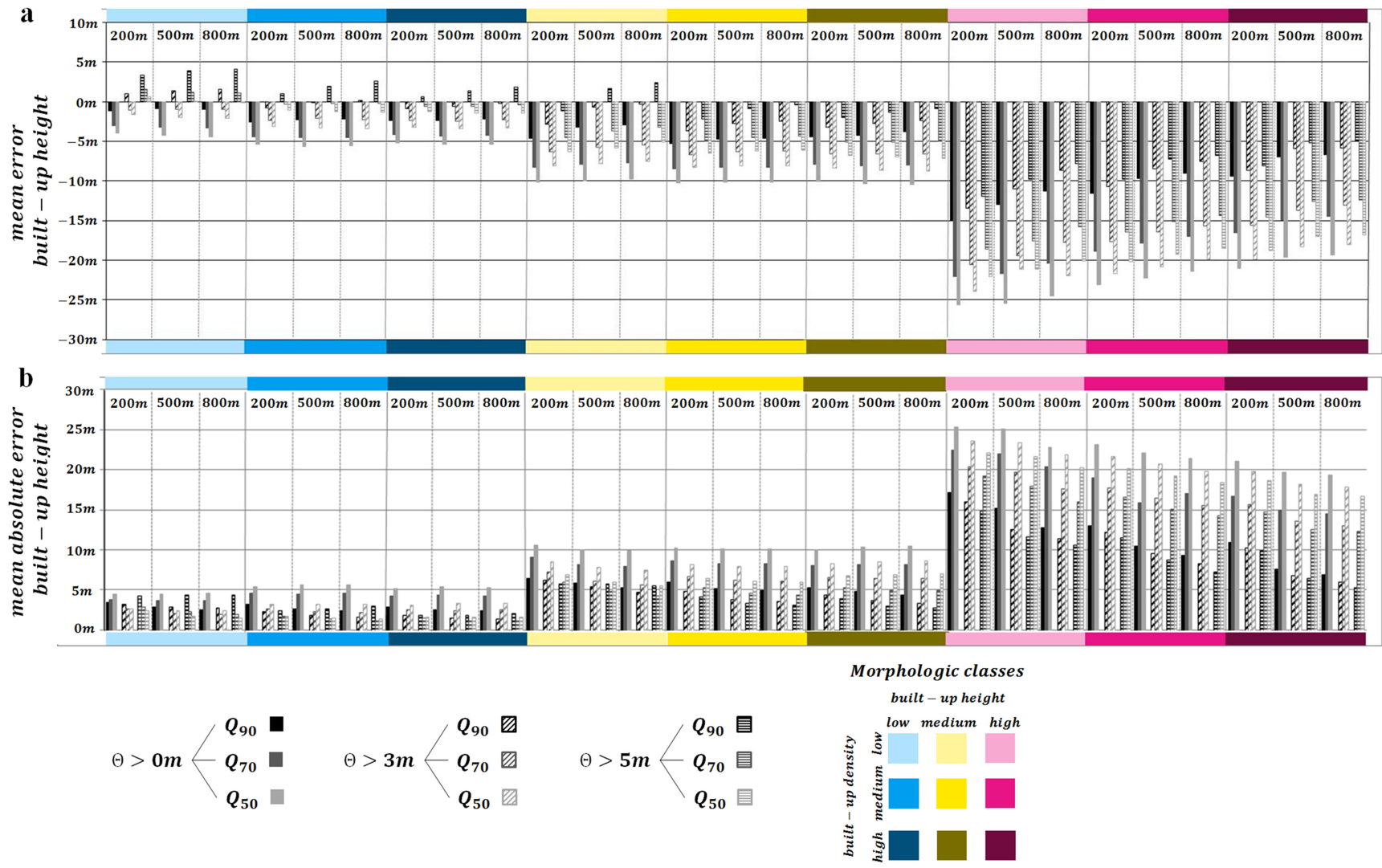

Fig. 5. Error measures for built-up height estimations. ME (a) and MAE (b) differentiated according to the nine morphologic classes and different values for height threshold $\Theta$, aggregation function for estimation of built-up height per grid cell, as well as size of the grid cells.

\section{A. Accuracy of Built-up Height}

Accuracy of built-up height estimations differentiated with respect to the nine morphologic classes is provided in Fig. $\mathbf{5}$. The morphologic classes were created based on the reference data sets of all 10 considered cities. Results are presented as a function of different values for height threshold $\Theta$, aggregation function for estimation of built-up height per grid cell, and size of the grid cells.
The ME reveals a systematic underestimation of built-up height estimations in relation to the reference data for all morphologic classes (Fig. 5a). Only some estimates based on restrictive values for $\Theta$ (i.e., $\Theta=\{>3 \mathrm{~m}, 5 \mathrm{~m}\}$ ) provide overestimations predominately for morphologic classes with low built-up density. The MAE (Fig. 5b) uncovers increasing height deviations with an increasing built-up height. This can be naturally related to the increasing range of numerical height values. In addition, areas which show a high built-up height in

\section{built-up height - LoD-1 model}

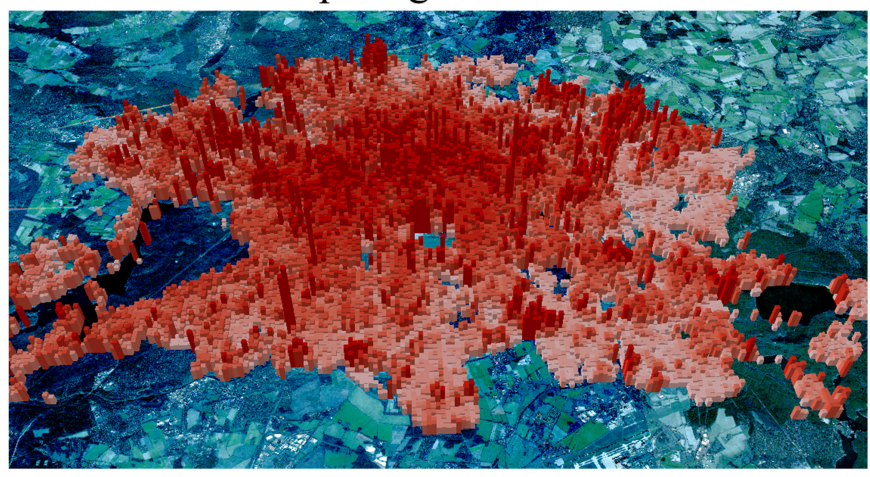

\section{BERLIN}
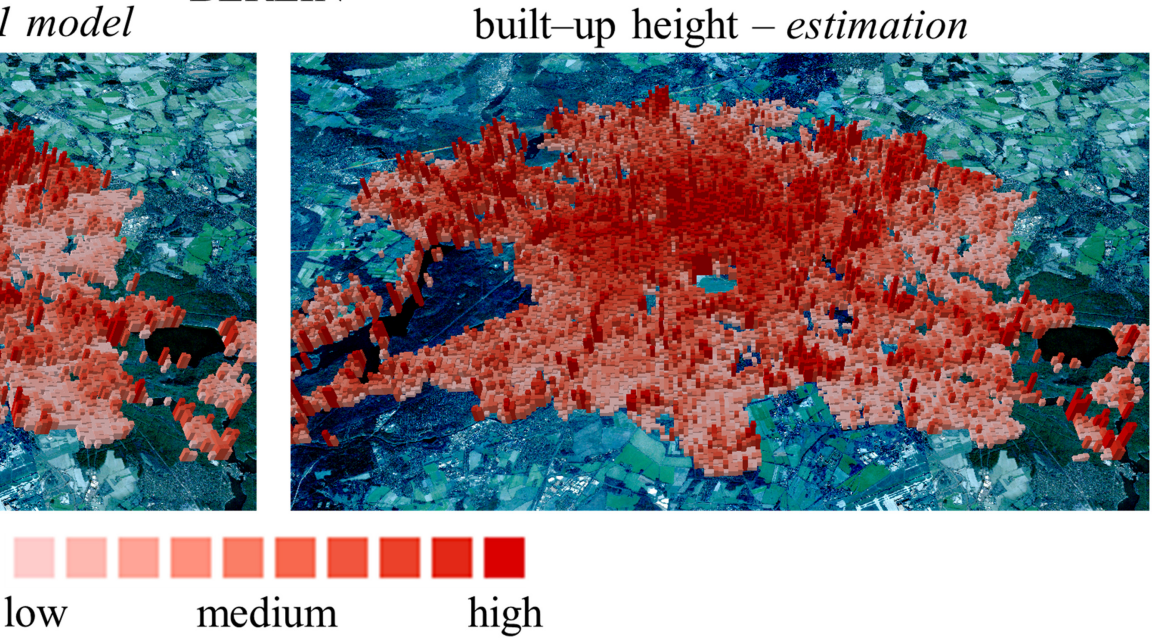

Fig. 6. Visualized built-up heights according to $a=200 \mathrm{~m}\left(\Theta=3 \mathrm{~m}\right.$ and height is represented by $Q_{90}$ with respect to the estimation) for the example of Berlin, Germany. Color coding corresponds to deciles for the individual data sets and thus allows a relative spatial comparison; however, heights of bars correspond to absolute numerical values where numerical 8 height values were superelevated 50 times. 

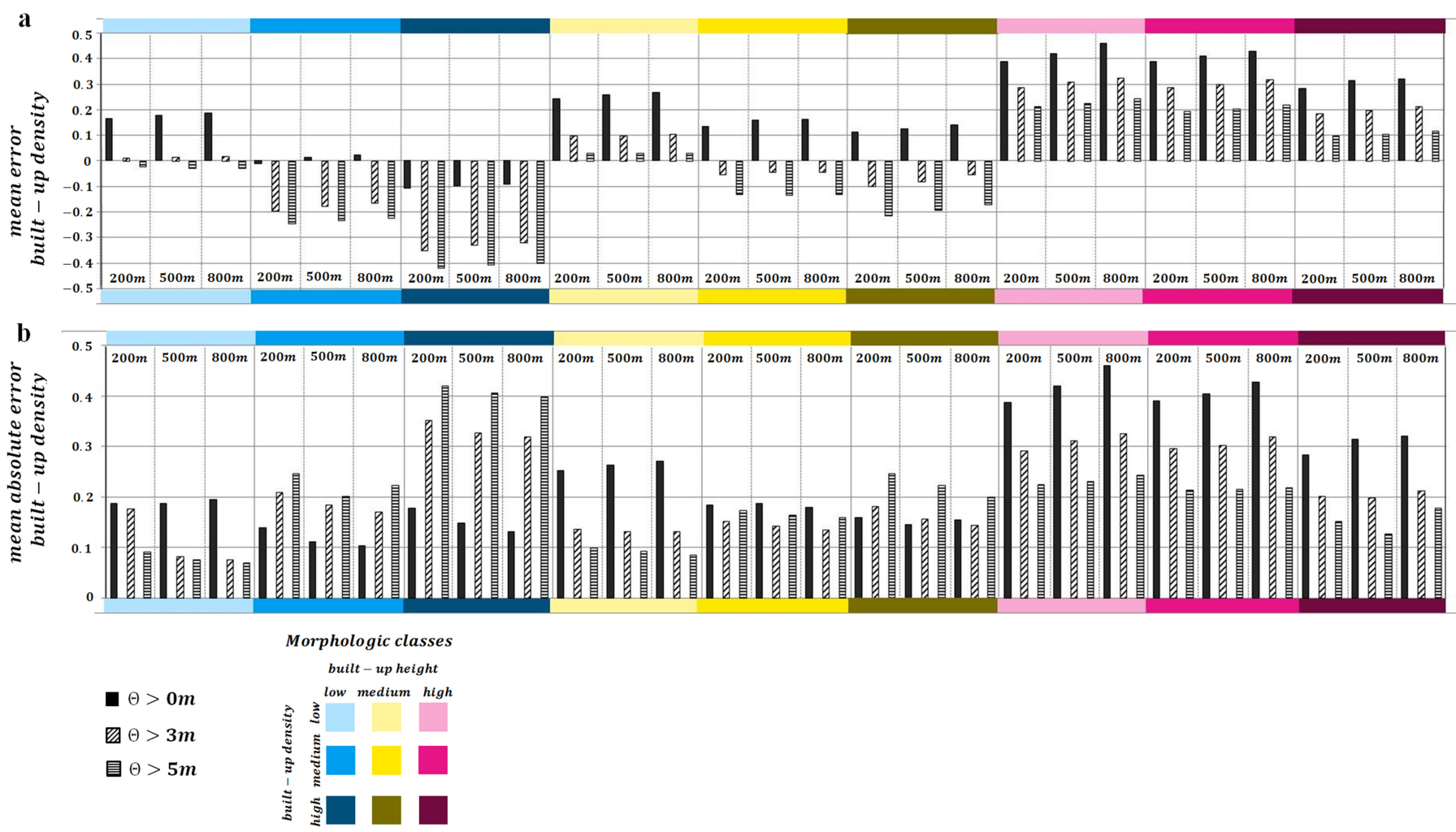

Fig. 7. Error measures for built-up density estimations. ME (a) and MAE (b) differentiated according to the nine morphologic classes and different values for height threshold $\Theta$, as well as size of the grid cells.

conjunction with a low built-up density are prone to layover and shadow effects, which prohibit capturing valid elevation measurements. However, height estimations feature lower error levels with an increasing built-up density. Generally, error levels can be balanced by choosing an appropriate aggregation function. Thereby, the most progressive value considered in this study (i.e., $Q_{90}$ ) consistently provides most favorable error levels, which underlines systematically low elevation values in the underlying TDM data in relation to the ground truth information. This relation also explains favorable error levels induced by restrictive $\Theta$ values. Thereby, only built-up areas are considered for height estimation, which already exceed certain elevation values, and, thus, provide progressive built-up height estimations. Overall, error levels based on favorable combinations of hyperparameters feature a deviation of less than one floor for built-up areas with low heights, 1-2 floors for medium high built-up areas, and approximately two floors for high built-up areas if built-up structures are not overly sparse, which underlines the viability of the approach. Finally, it can be noted that errors levels frequently decrease slightly with an increasing size of the grid cells, which can be related to averaging effects.

To illustrate further, Fig. 6 provides a visual comparison of estimated built-up heights and built-up heights derived from the building geometries of the LoD-1 model for the city of Berlin, Germany. In concordance with the previous results, it can be noted that estimated built-up heights are in tendency underestimated. However, the relative spatial pattern reflects the distribution of built-up heights with respect to the reference data set well. For instance, the urban core with high built-up heights is equally well depicted as low built-up heights on the peripheral areas of the city.

\section{B. Accuracy of Built-up Density}

Accuracy of built-up density estimations differentiated according to the nine morphologic classes is provided in Fig. 7. Analogous to previous analysis, they are based on the reference data sets of all 10 considered cities. Results are presented as a function of different values for height threshold $\Theta$ and size of the grid cells.

ME values (Fig. 7a) show varying under- and overestimations with respect to the morphologic classes. Overestimations can be observed predominantly for areas which are characterized by high built-up heights. Those builtup structures can be primarily found in the central areas of the considered cities (i.e., the urban cores). Here, the estimations reveal an almost complete coverage by built-up structures while the reference data depict significant lower built-up densities. In contrast to that, underestimations can be noted mainly for areas which feature low built-up heights. Those built-up structures can be frequently found in the fringe of the agglomerations (i.e., residential areas at peripheral or suburban areas of the cities). The estimations assign very low built-up densities to those areas while the cadastral data, which is considered as ground truth information here, reveal significantly higher built-up densities. This relation can be dominantly attributed to the fact that intra-urban vegetation obscures underlying built-up structures when corresponding built-up heights are low. Comparable effects were also previously described in studies which aim to estimate the 


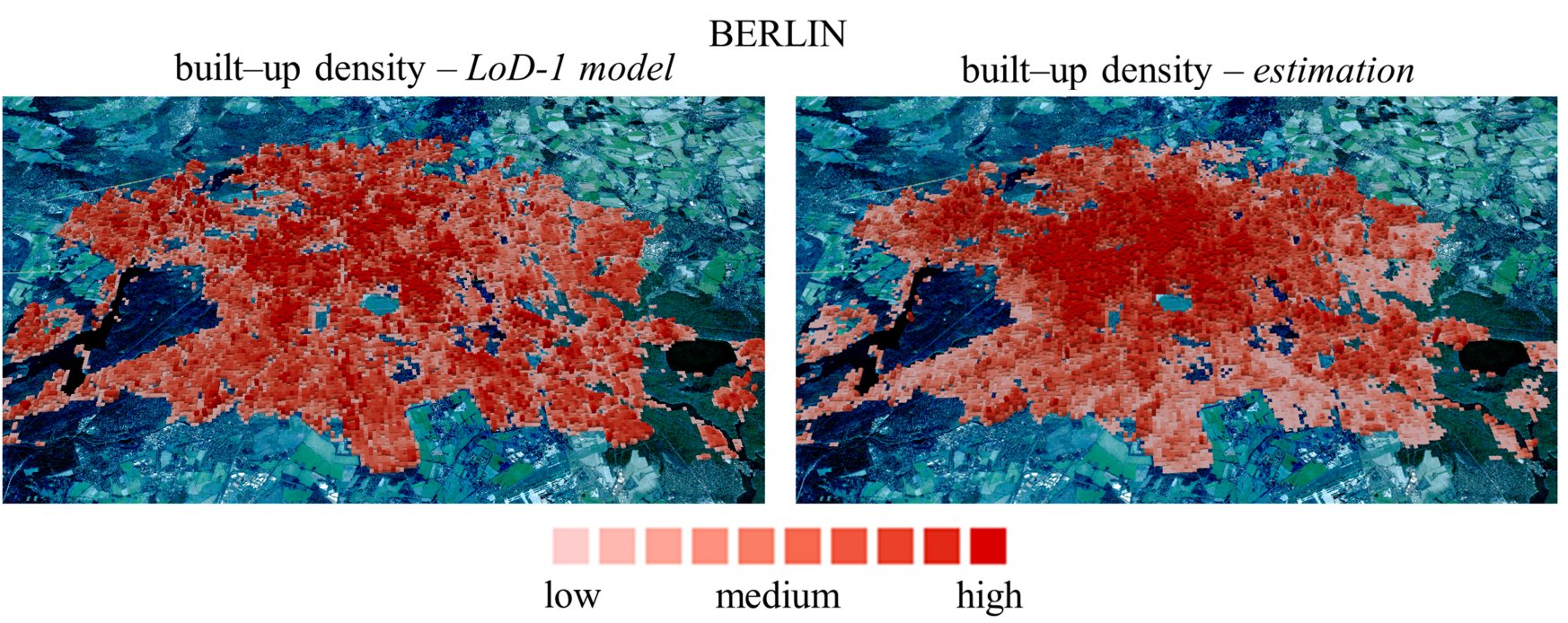

Fig. 8. Visualized built-up densities according to $a=200 \mathrm{~m}(\Theta=3 \mathrm{~m}$ with respect to the estimation) for the example of Berlin, Germany. Color coding corresponds to deciles for the individual data sets and thus allows a relative spatial comparison; however, heights of bars correspond to absolute numerical values where numerical density values were superelevated 1000 times.

percentage of impervious surfaces based on remote sensing imagery (e.g., [52], [60]). Besides, for remaining morphologic classes, overestimations for progressive $\Theta$ values and underestimations for restrictive $\Theta$ values occur.

Regarding absolute error levels and in concordance with previous results, the MAE (Fig. 7b) reveals lowest values for areas with medium built-up heights and largest values for structures with high built-up heights and low built-up densities (i.e., overestimations regarding the urban cores) as well as for areas with low built-up heights and high built-up densities (i.e., underestimations regarding residential areas at peripheral and suburban areas of the cities). Thereby, also most favorable hyperparameters vary as a function of morphologic class: progressive $\Theta$ values allow for reducing underestimations in areas with low built-up height and high built-up density, while restrictive $\Theta$ values enable balancing overestimations for structures with high built-up height and low built-up density. However, for a moderately restrictive $\Theta$ threshold (i.e., $\Theta=\{>3 \mathrm{~m}\}$ ), viable built-up density estimations with deviations less than $20 \%$ are achieved for the majority of morphologic classes. An additional $10 \%$ deviation can be observed for the morphologic classes comprising high built-up heights and low and medium built-up densities as well as the class with low built-up heights and high built-up densities. In this setting and analogous to the built-up height estimations, errors levels frequently decrease slightly with an increasing size of the grid cells, since larger grid sizes are more likely to contain a mixture of structural types leveling local variations of density throughout urban morphology.

To illustrate the previous findings, Fig. 8 provides a visual comparison of estimated built-up densities and built-up densities derived from the building geometries of the LoD-1 model for the city of Berlin, Germany. The density estimations show an ideal decrease from the core to the fringe, whereby the LoD-1 model-based built-up densities also show highest densities in the urban core but are generally more spatially differentiated and fragmented and feature higher levels in vast parts of the fringe areas.

\section{Morphologic Characterization}

The built-up height and density estimations are finally combined within nine morphologic classes. Results for the capital cities are depicted in Fig. 9 with affiliated classification accuracy measures computed by comparing every single grid cell. Additionally, zoom-in windows for selected areas are shown. Results for the remaining cities are visualized in Fig. 10 with affiliated classification accuracy measures.

First it can be noted that the overall morphologic structure of the cities is well reflected: They are dominantly characterized by a single or multiple urban core(s) and affiliated high built-up structures and lower built-up structures, which are also less densely arranged, in the peripheral parts. This structure primarily derives from a concentric, industrial city model with a defined center surrounded by a more or less complex halo of settlement structures with lower heights and densities and suburbs which ultimately turn into rural environments [61]. Thereby, smallscale changes in the urban morphology are well reflected as can be seen from the classification outcome which is superimposed on the Sentinel-2 imagery in the zoom-inwindows of Fig. 9.

Regarding the class-specific classification accuracy measures, the user's and producer's accuracies reflect the findings from the previous analysis. While certain morphologic classes feature consistently both high user's and producer's accuracies, other morphologic classes mirror characteristic errors described in the evaluation of estimated built-up heights and densities before. Large omission errors (i.e., low producer's accuracies) can be observed for the class characterizing low built-up heights and high built-up densities with respect to the majority of cities (i.e., Berlin, London, Munich, Frankfurt, Stuttgart, The Hague). Here, the underestimation of built-up densities in fringe areas prevents 
correct class assignments and corresponding areas are primarily allocated to the class with low built-up height and low built-up density. Likewise, large omission errors can be noticed for majority of cities for the two classes characterizing high built-up heights and low and medium built-up densities. Here, the general underestimation of built-up heights causes incorrect class assignments and corresponding areas are primarily allocated to the classes with medium built-up heights. Besides, some local sources of errors can be identified. As such, erroneous class assignments are present in cities where built-up structures in direct spatial vicinity to large water bodies exist (e.g., Amsterdam and Rotterdam). Although water bodies were pruned from further processing by inclusion of the TDM water mask (cf. section III-D), a number of pixels still carry disproportional high height values in the UF-E model that lead to errors of commission for the class which describes high built-up heights and high and medium built-up densities. Nevertheless, global classification accuracy measures show OA values consistently exceeding $70 \%$ and $\kappa$ statistics dominantly show substantial agreements (i.e., $\kappa$ statistic $>0.6$ ), which underlines the viability of the approach. As such, the produced data sets feature a great potential for novel applications and objective comparative analysis between cities regarding the morphologic structure based on data with an unprecedented tradeoff between high spatial resolution and large-area coverage. 

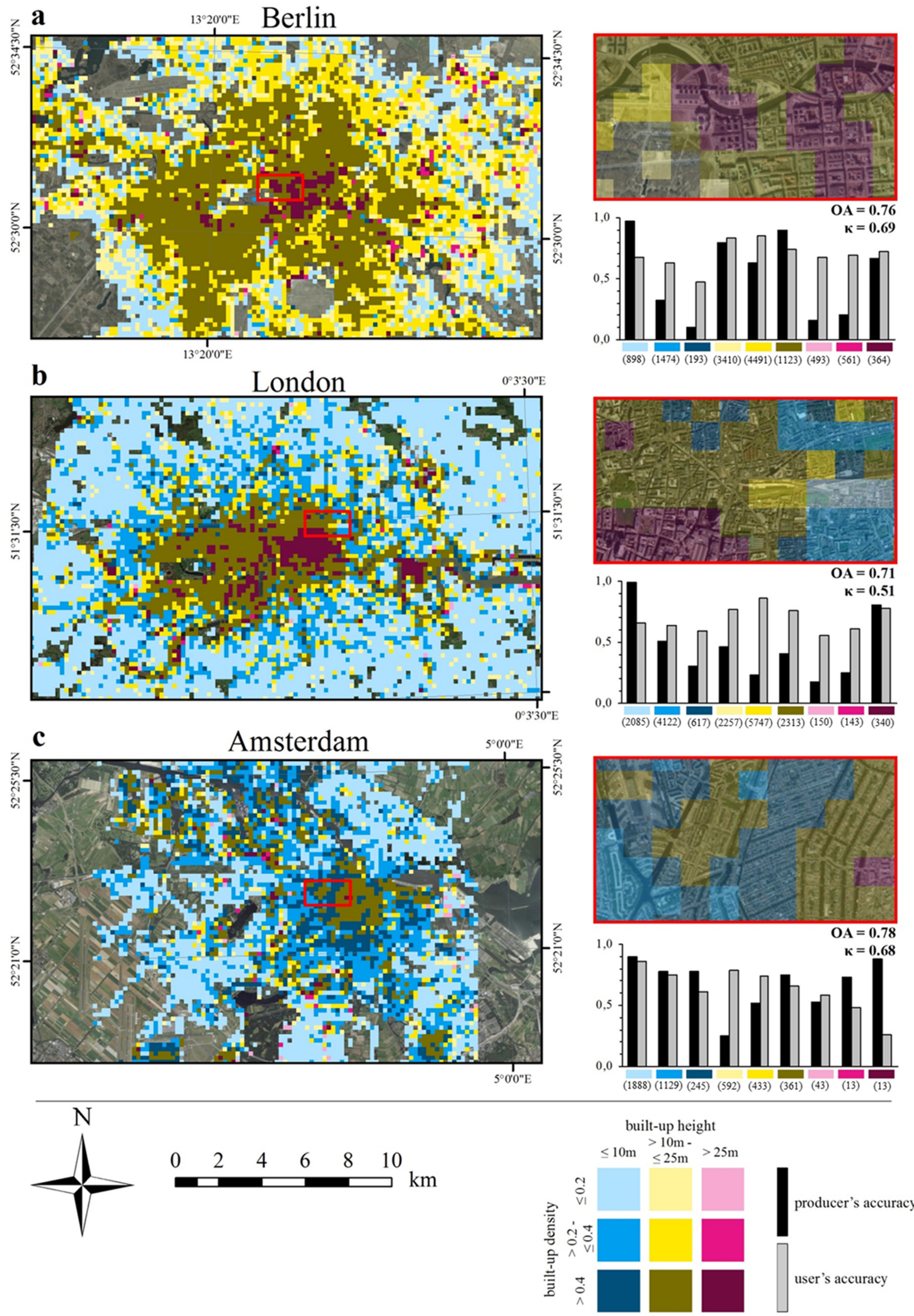

Fig. 9. Morphologic characterization for the capital cities Berlin (a), London (b), and Amsterdam (c) according to $a=$ $200 \mathrm{~m}$; semi-transparent detailed views are superimposed on Sentinel-2 imagery. Ordinally weighted classification accuracy measures are also presented. Numbers in brackets below the producer's and user's accuracy bars indicate the numbers of grid cells per morphologic class based on the reference data which were used for computation of accuracy measures. 

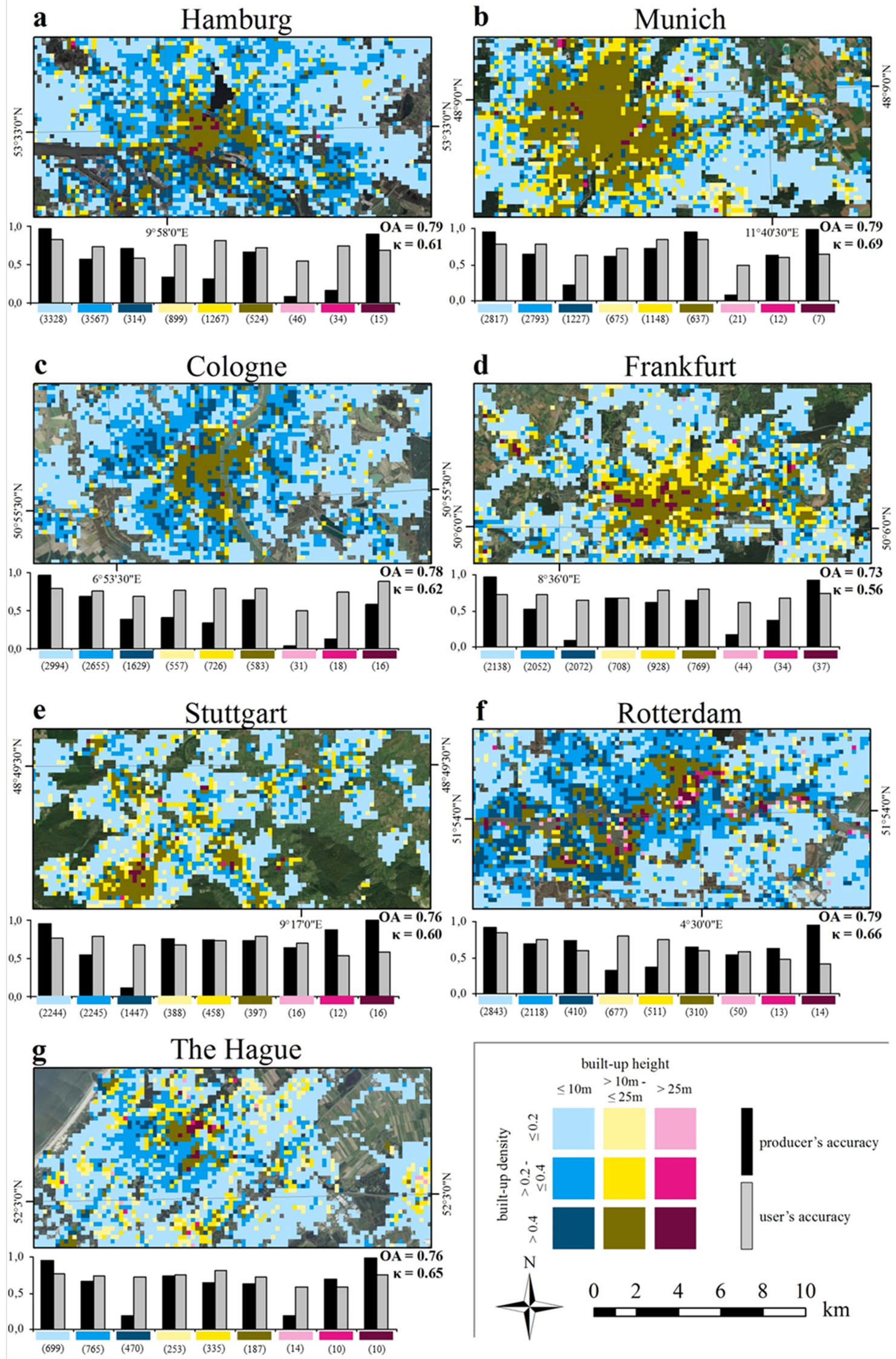

Fig. 10. Morphologic characterization for the cities a) Hamburg, b) Munich, c) Cologne, d) Frankfurt, e) Stuttgart, f) Rotterdam, and g) The Hague according to $a=200 \mathrm{~m}$. Ordinally weighted classification accuracy measures are also presented. Numbers in brackets below the producer's and user's accuracy bars indicate the numbers of grid cells per 3 morphologic class based on the reference data which were used for computation of accuracy measures. 


\section{CONCLUSIONS AND OUTLOOK}

In this paper, we have proposed a novel workflow, which builds upon data from the TDM and Sentinel-2 imagery to characterize urban morphology for large areas in an automated manner. To this purpose, we rely on the GUF processor, which discriminates between "built-up" and "non built-up" LC on a global scale while maintaining a high spatial detail. This binary description of built-up LC is extended by computing elevation information (i.e., UF-E model) from the TDM using a tailored filtering technique on the DSM. Derived information layers serve as input for a hierarchical classification scheme for intra-urban areas. The scheme foresees the delineation of intra-urban vegetation under consideration of information from the Sentinel-2 imagery. Subsequent to that, the UF-E model is used to further distinguish between "elevated builtup" and "residual intra-urban LC" in the remaining areas. Consequently, pixels of the class "elevated built-up" and affiliated elevation values from the UF-E model are used for mapping of built-up densities and heights and for creating the joint classes, which describe urban morphology.

Comparative evaluations with respect to cadastral data are obtained for settlement areas covering 10 major cities in Germany, England, and the Netherlands. They uncover a systematic underestimation of built-up heights in relation to the reference data for all morphologic classes. However, error levels can be balanced by choosing appropriate hyperparameters of the approach. In this manner, built-up height estimations feature deviations of approximately 1-2 floors depending on the corresponding morphologic class and the relative spatial pattern reflects the distribution of built-up heights with respect to the reference data set well. The built-up densities show highest accuracies for areas with medium builtup heights. Lowest accuracies are obtained for structures with high built-up heights and low built-up densities (i.e., overestimations regarding the intra-urban cores) as well as for areas with low built-up heights and high built-up densities (i.e., underestimations regarding peripheral or suburban areas of the cities). Nevertheless, viable built-up density estimations with deviations of less than $20 \%$ are achieved for the majority of morphologic classes. In addition, the estimations reflect the general urban morphologic structure well by showing an ideal decrease from the core to the fringe of the agglomeration areas. Finally, regarding the actual morphologic characterization, ordinally weighted global classification accuracy measures show OA values consistently exceeding $70 \%$ and $\kappa$ statistics dominantly show substantial agreements.

The TDM and Sentinel-2 data offer the unique opportunity to assess urban morphology according to the proposed approach around the globe. However, for scientific applications the accessibility of the TDM data is currently limited to $100000 \mathrm{~km}^{2}$ per data proposal. To alleviate this limited accessibility, we aim to develop an approach to substitute the TDM data. To this purpose, we will render the estimation of built-up density and height as a supervised learning problem [62]. In addition to that, we aim for reprocessing the Co-registered Single-look Slant-range Complex TDM data to establish a novel global DSM with enhanced geometric resolution properties using advanced signal processing algorithms [63], [64]. Thereby, future work can also aim for refining accuracy of results by establishing empirically-derived postprocessing models to align the estimations to reference data. This appears as a viable option since we found majority of deviations to be systematic (i.e., data inherent) and thus allow to be corrected for.

Overall, the envisaged data sets have the potential to substantially support and enable a broad range of area-wide applications as discussed in the introduction section. Besides, the TDM has been extended to generate an updated elevation model [49]. With it, monitoring of urban expansion could be extended from two-dimensional analysis [65] towards the quantification of change of built-up volumes over time. For the very first time, the extent of infill development and urban intensification would become observable for larger urban areas or even urban systems at national or supranational scales. Hence, valuable contributions to different fields of research are in prospect.

\section{APPENDIX A}

\section{TABLE I \\ LIST OF ABBREVIATIONS}

DSM digital surface model

DTM digital terrain model

EO earth observation

GUF Global Urban Footprint

LC land cover

LCZ local climate zone

LoD-1 level of detail 1

ME mean error

MAE mean absolute error

NDSM normalized DSM

NDVI Normalized Difference Vegetation Index

OA overall accuracy

SAR synthetic aperture radar

TDM TanDEM-X mission

UF-E TanDEM-X mission

VHR very high spatial resolution

\section{ACKNOWLEDGEMENTS}

This work was supported by the German Federal Ministry for Economic Affairs and Energy's initiative "Smart Datainnovations from data" under grant agreement: "smart data for catastrophe management (sd-kama, 01MD15008B)". The work of Christian Geiß was supported by the Helmholtz Association under the grant "pre_DICT" (PD-305). The authors also would like to thank the German Research Foundation (DFG) for financing the research project "Where are the jobs? Stadtregionale Zentrenstrukturen im internationalen Vergleich" with the grant number: TA 800/6-1 $\&$ SI 932/12-1. This work has also received funding from the European Research Council (ERC) under the European Union's Horizon 2020 research and innovation programme (grant agreement No [714087] - So2Sat). Additionally, this research was funded in part by the BMBF, grant no. 03G0876. 


\section{REFERENCES}

[1] T. Leichtle, C. Geiß, M. Wurm, T. Lakes, and H. Taubenböck, "Unsupervised change detection in VHR remote sensing imagery - an objectbased clustering approach in a dynamic urban environment, "International Journal of Applied Earth Observation and Geoinformation, vol. 54, pp. 15-27, 2017.

[2] X. Huang, D. Wen, J. Li, and R. Qin, "Multi-level monitoring of subtle urban changes for the megacities of China using high-resolution multi-view satellite imagery," Remote Sens. Environ., vol. 196, pp. 56-75, 2017.

[3] J. Graesser et al., "Image based characterization of formal and informal neighborhoods in an urban landscape," IEEE J. Sel. Topics Appl. Earth Observ. Remote Sens., vol. 5, no. 4, pp. 1164-1176, Aug. 2012.

[4] H. Taubenböck et al., "Delineation of central business districts in mega city regions using remotely sensed data," Remote Sens. Environ., vol. 136, pp. 386-401, Sep. 2013.

[5] S. Wu, X. Qiu, and L. Wang, "Population Estimation Methods in GIS and Remote Sensing: A Review," International Journal of Remote Sensing, vol. 42, no. 1, pp. 80-96, 2005.

[6] C. Geiß et al., "Joint use of remote sensing data and volunteered geographic information for exposure estimation - evidence from Valparaíso, Chile," Natural Hazards, vol. 86, pp. 81-105, 2017.

[7] P. Rode, C. Keim, G. Robazza, P. Viejo, and J. Schofield, "Cities and energy: urban morphology and residential heat-energy demand," Environment and Planning B: Planning and Design, vol. 41, pp. 138-162, 2014.

[8] M. Silva, V. Oliveira and V. Leal, "Urban form and energy demand: A review of energy-relevant urban attributes," Journal of Planning Literature, vol. 32 , pp. $346-365,2017$

[9] C. Geiß et al., "Remote sensing-based characterization of settlement structures for assessing local potential of district heat," Remote Sens., vol. 3, no. 7, pp. 1447-1471, 2011.

[10] T. R. Tooke et al., "Mapping demand for residential building thermal energy services using airborne LiDAR," Applied Energy, vol. 127, pp. 125134,2014

[11] D. Godoy-Shimizu, P. Steadman, I. Hamilton, M. Donn, S. Evans, G. Moreno and H. Shayesteh, "Energy use and height in office buildings," Building Research \& Information, online first, 2018.

[12] C. Geiß and H. Taubenböck, "Remote sensing contributing to assess earthquake risk: From a literature review towards a roadmap," Natural Hazards, vol. 68, no. 1, pp. 7-48, Aug. 2013.

[13] M. Pittore, M. Wieland, and K. Fleming, "Perspectives on global dynamic exposure modelling for geo-risk assessment," Natural Hazards, vol. 86, pp. 7-30, 2017.

[14] B. Stone Jr. and M.O. Rodgers, "Urban form and thermal efficiency: how the design of cities influences the urban heat island effect," Journal of the American Planning Association, vol. 67, no. 2, pp. 186-198, 2001.

[15] R. Watkins, J. Palmer J, and M. Kolokotroni, "Increased temperature and intensification of the urban heat island: implications for human comfort and urban design," Built Environment, vol. 33, pp. 85-96, 2007.

[16] J. Heinzel and T. Kemper, "Automatic metric characterization of urban structure using building decomposition from very high resolution imagery," International Journal of Applied Earth Observation and Geoinformation, vol. 35, pp. 151-160, 2015.

[17] D. Gonzáles-Aguilera et al., "Automated Urban Analysis Based on LiDAR-Derived Building Models," IEEE Trans. Geosci. Remote Sens., vol. 51, no. 3, pp. 1844-1851, Mar. 2013.

[18] H. Taubenböck et al., "The morphology of the Arrival City - A global categorization based on literature surveys and remotely sensed data," Appl. Geography, vol. 92, pp. 150-167, 2018.

[19] G. Krieger et al., "TanDEM-X: A satellite formation for high-resolution SAR interferometry," IEEE Trans. Geosci. Remote Sens., vol. 45, no. 11, pp. 3317-3341, Nov. 2007.

[20] M. Zink et al., "TanDEM-X: the new global DEM takes shape," IEEE Geosci. Remote Sens. Mag., vol. 2, no. 2, pp. 8-23, 2014.

[21] M. Drusch et al., "Sentinel-2: ESA's Optical High-Resolution Mission for GMES Operational Services," Remote Sens. Environ., vol. 120, pp. 25-36, May 2012.

[22] C. Geiß et al., "Assessment of seismic building vulnerability from space," Earthquake Spectra, vol. 30, no. 4, pp. 1553-1584, Nov. 2014.

[23] M. Belgiu, I. Tomljenovic, T. Lampoltshammer, T. Blaschke, and B. Höfle, "Ontology-based classification of building types detected from airborne laser scanning data," Remote Sens., vol. 6, no. 2, pp. 1347-1366, Feb. 2014
[24] S. Du et al., "Semantic classification of urban buildings combining VHR image and GIS data: An improved random forest approach," ISPRS J. Photogramm. Remote Sens., vol. 105, pp. 107-119, 2015.

[25] C. Geiß et al., "Estimation of seismic buildings structural types using multi-sensor remote sensing and machine learning techniques," ISPRS J. Photogramm. Remote Sens., vol. 104, pp. 175-188, 2015.

[26] M. Wurm, A. Schmitt, and H. Taubenböck, "Building Types" Classification Using Shape-Based Features and Linear Discriminant Functions," IEEE J. Sel. Topics Appl. Earth Observ. Remote Sens., vol. 9, no 5, pp. 1901-1912, May 2016.

[27] C. Geiß et al., "Multitask Active Learning for Characterization of Built Environments with Multisensor Earth Observation Data," IEEE J. Sel. Topics Appl. Earth Observ. Remote Sens., vol. 10, no. 12, pp. 5583-5597, 2017.

[28] U. Heiden, W. Heldens, S. Roessner, K. Segl, T. Esch, and A. Mueller, "Urban structure type characterization using hyperspectral remote sensing and height information," Landsc. Urban Plann., vol. 105, pp. 361-375, 2012.

[29] C. Geiß, M. Jilge, T. Lakes, and H. Taubenböck, "Estimation of Seismic Vulnerability Levels of Urban Structures With Multisensor Remote Sensing," IEEE J. Sel. Topics Appl. Earth Observ. Remote Sens., vol. 9, no 5, pp. 19131936, May 2016.

[30] P. Soille, Morphological Image Analysis: Principles and Applications. Springer, 2004.

[31] T. Zhang, X. Huang, D. Wen, and J. Li, "Urban Building Density Estimation From High-Resolution Imagery Using Multiple Features and Support Vector Regression," IEEE J. Sel. Topics Appl. Earth Observ. Remote Sens., vol. 10, no 7, pp. 3265-3280, Jul 2017.

[32] D. Luebke, B. Watson, J.D. Cohen, M. Reddy, and A. Varshney, Level of Detail for 3D Graphics. Elsevier Science Inc., 2002.

[33] A. Krehl, S. Siedentop, H. Taubenböck, and M. Wurm, "A comprehensive view on urban spatial structure: urban density patterns of German city regions" International Journal of Geo-Information, vol. 5, pp. 121, 2016.

[34] H. Taubenböck et al., "The Physical Density of the City - Deconstruction o the Delusive Density Measure with Evidence from Two European Megacities," ISPRS Int. J. Geo-Inf., vol. 5, no. 11, 2016.

[35] C. Berger, M. Voltersen, R. Eckardt, J. Eberle, T. Heyer, N. Salepci, S. Hese, C. Schmullius et al., "Multi-modal and multi-temporal data fusion: Outcome of the 2012 GRSS data fusion contest," IEEE J. Sel. Topics Appl. Earth Observations Remote Sens., vol. 6, no. 3, pp. 1324-1340, Jun. 2013.

[36] I. D. Stewart and T. R. Oke, "Local climate zones for urban temperature studies," Bull. Amer. Meteorol. Soc., vol. 93, no. 12, pp. 1879-1900, Dec. 2012.

[37] B. Bechtel and C. Daneke, "Classification of local climate zones based on multiple earth observation data," IEEE J. Sel. Topics Appl. Earth Observ. Remote Sens., vol. 5, no. 4, pp. 1191-1202, Aug. 2012.

[38] B. Bechtel, "Mapping Local Climate Zones for a Worldwide Database of the Form and Function of Cities," ISPRS International Journal of GeoInformation, vol. 4, pp. 199-219, 2015.

[39] C. Geiß, M. Wurm, and H. Taubenböck, "Towards large-area morphologic characterization of urban environments using the TanDEM-X mission and Sentinel-2," 2017 Joint Urban Remote Sensing Event (JURSE), Dubai, United Arab Emirates, pp. 1-4, 2017. doi: 10.1109/JURSE.2017.7924543.

[40] T. Esch et al., "Breaking new ground in human settlements from space The Global Urban Footprint," ISPRS Journal of Photogrammetry and Remote Sensing, vol. 134, pp. 30-42, 2017.

[41] M. Klotz, T. Kemper, C. Geiß, T. Esch, and H. Taubenböck, "How good is the map? A multi-scale cross-comparison framework for global settlement layers: Evidence from Central Europe," Remote Sens. Environ., vol. 178, pp. 191-212, 2016

[42] C. Geiß, M. Wurm, M. Breunig, A. Felbier, and H. Taubenböck, "Normalization of TanDEM-X DSM data in urban environments with morphological filters," IEEE Trans. Geosci. Remote Sens., vol. 53, no. 8, pp. 4348-4362, Aug. 2015.

[43] B. Wessel, "TanDEM-X Ground Segment - DEM Products Specification Document”, EOC, DLR, Oberpfaffenhofen, Germany, Public Document TDGS-PS-0021, Issue 3.1, 2016. [Online]. Available: https://tandemxscience.dlr.de/

[44] J. W. Rouse, R. H. Haas, J. A. Schell, and D. W. Deering, "Monitoring vegetation systems in the great plains with ERTS," in Proc. 3rd Earth Resour. Technol. Satell.-1 Symp., 1974, pp. 309-317

[45] J. Schreyer, C. Geiß, and T. Lakes, "TanDEM;-X for Large-Area Modeling of Urban Vegetation Height: Evidence from Berlin, Germany," 
IEEE J. Sel. Topics Appl. Earth Observ. Remote Sens., vol. 9, no 5, pp. 18761887, May 2016.

[46] T. Esch, M. Thiel, A. Schenk, A. Roth, A. Müller, and S. Dech, "Delineation of urban footprints from TerraSAR-X data by analyzing speckle characteristics and intensity information," IEEE Trans. Geosci. Remote Sens., vol. 48, no. 2, pp. 905-916, Feb. 2010.

[47] N. Otsu, "A Threshold Selection Method from Gray-Level Histograms," IEEE Trans. Systems, Man, Cybernetics, vol. SMC-9, no. 1, pp. 62-66, Jan. 1979.

[48] B. Wessel et al., "Accuracy assessment of the global TanDEM-X Digital Elevation Model with GPS data," ISPRS J. Photogramm. Remote Sens., vol. 139, pp. 171-182, 2018.

[49] P. Rizzoli et al., "Generation and performance assessment of the global TanDEM-X digital elevation model," ISPRS J. Photogramm. Remote Sens., vol. 132, pp. 119-139, 2017.

[50] European Space Agency (ESA) - Science Toolbox Exploitation Platform, Sentinel Application Platform; URL: http://step.esa.int/main/toolboxes/snap/ Last accessed: 16 May 2018a.

[51] European Space Agency (ESA) - Sen2Cor; URL: http://step.esa.int/main/third-party-plugins-2/sen2cor/ Last accessed: 16 May $2018 \mathrm{~b}$.

[52] P. Leinenkugel, T. Esch, and C. Künzer, "Settlement detection and impervious surface estimation in the Mekong Delta using optical and SAR remote sensing data," Remote Sens. Environ., vol. 115, pp. 3007-3019, 2011.

[53] M. Wurm, P. d'Angelo, P. Reinartz, and H. Taubenböck, "Investigating the Applicability of Cartosat-1 DEMs and Topographic Maps to Localize Large-Area Urban Mass Concentrations," IEEE J. Sel. Topics Appl. Earth Observ. Remote Sens., vol. 7, no 10, pp. 4138-4152, Oct. 2014.

[54] ESRI Nederland - Community Map Contributors. URL: http://www.arcgis.com/apps/Viewer/index.html?

appid=2e4c2b5b127a447e94997bbffd41b93e Last accessed: 16 May 2018.

[55] D. Shepard, "A two-dimensional interpolation function for irregularly spaced data," in Proc. 23rd ACM Nat. Conf. (ACM'68), Aug. 27-29, 1968, pp. 517-524.

[56] C. J. Willmott and K. Matsuura, "Advantages of the mean absolute error (MAE) over the root mean square error (RMSE) in assessing average model performance," Clim. Res., vol. 30, pp. 79-82, 2005.

[57] J. S. Cardoso and R. Sousa, "Measuring the Performance of Ordinal Classification," International Journal of Pattern Recognition and Artificial Intelligence, vol. 25, no. 8, pp. 1173-1195, 2011.

[58] G. M. Foody, "Thematic map comparison: Evaluating the statistical significance of differences in classification accuracy," Photogramm. Eng. Remote Sens., vol. 50, no. 5, pp. 627-633, 2004.

[59] M.Wurm, H. Taubenböck, M. Schardt, T. Esch, and S. Dech, "Objectbased image information fusion using multisensor earth observation data over urban areas," International Journal of Image and Data Fusion, vol. 2, no. 2, pp. 121-147, 2011.

[60] T. Esch, V. Himmler, G. Schorcht, M. Thiel, T. Wehrmann, F. Bachofer, C. Conrad, M. Schmidt, and S. Dech, "Large-area assessment of impervious surface based on integrated analysis of single-date Landsat-7 images and geospatial vector data," Remote Sens. Environ., vol. 113, pp. 1678-1690, 2009.

[61] H. Taubenböck, I. Standfuß, M. Wurm, A. Krehl, and S. Siedentop, "Measuring morphological polycentricity - A comparative analysis of urban mass concentrations using remote sensing data," Computers, Environment and Urban Systems, vol. 64, pp. 42-56, 2017.

[62] C. Geiß, H., Schrade, and H., Taubenböck, "Boosted Machine Learning Ensemble Regression with Decision Fusion Strategy for Mapping Built-up Height and Built-up Density with OpenStreetMap Data and Sentinel-2 Imagery," 5th EARSeL Joint Workshop "Urban Remote Sensing - Challenges \& Solutions ", 24-26 September 2018, Bochum, Germany.

[63] G. Baier et al., "A Nonlocal InSAR Filter for High-Resolution DEM Generation From TanDEM-X Interferograms," IEEE Trans. Geosci. Remote Sens., in press, 2018

[64] X. X. Zhu, G. Baier, M. Lachaise, Y. Shi, F. Adam, and R. Bamler, "Potential and limits of non-local means InSAR filtering for TANDEM-X high-resolution DEM generation," Remote Sens. Environ., vol. 218, pp. 148$161,2018$.

[65] H. Taubenböck, T. Esch, A. Felbier, M. Wiesner, A. Roth, and S. Dech, "Monitoring urbanization in mega cities from space," Remote Sens. Environ., vol. 117, pp. 162-176, 2012. 